\title{
Searches for Magnetic Monopoles: A Review ${ }^{\dagger}$
}

\author{
Vasiliki A. Mitsou (D) \\ Instituto de Física Corpuscular (IFIC), CSIC—Universitat de València, C/ Catedrático José Beltrán 2, \\ E-46980 Paterna (Valencia), Spain; vasiliki.mitsou@ific.uv.es; Tel.: +34-963-543-855 \\ + Presented at the 7th International Conference on New Frontiers in Physics (ICNFP 2018), \\ Crete, Greece, 4-12 July 2018.
}

Published: 12 August 2019

check for updates

\begin{abstract}
This report briefly reviews selected searches for magnetic monopoles. The theoretical motivation behind their existence is highlighted. The focus is on the results of the searches and the bounds set in cosmic and collider detectors, especially in the current experiments operating at the Large Hadron Collider: ATLAS and MoEDAL.
\end{abstract}

Keywords: magnetic monopoles; LHC; MoEDAL; ATLAS

\section{Theoretical Motivation}

The main theoretical motivations behind the hypothetical existence of magnetic monopoles are the symmetrisation of the Maxwell's equations and the explanation of the charge quantisation. Dirac [1] proved that magnetic monopoles could explain the discrete nature of the electric charge, leading to the Dirac Quantisation Condition (DQC),

$$
\alpha g=\frac{N}{2} e, \quad N=1,2, \ldots,
$$

where $\alpha=\frac{e^{2}}{4 \pi \varepsilon_{0}}=\frac{1}{137}$ is the fine structure constant, $e$ is the electron charge, $\varepsilon_{0}$ is the vacuum permittivity, and $g$ is the monopole magnetic charge in units $\hbar=c=1$. This quantisation condition should be modified by a factor of three if quarks existed free in Nature. The monopole mass and spin are not determined and are free parameters of the theory. This attractive proposal has revived a number of experimental investigations since then, with some of them briefly highlighted in this conference report.

The existence of magnetic monopoles, characterised by their isolated magnetic charges similar to electrically-charged particles, has been assumed over the years in many theoretical proposals [1-18]. On the other hand, dyons, carrying both magnetic and electric charge, offer a more involved solution leading to the DQC, which depends on the underlying theoretical scenario. Some of the theoretical scenarios predicting the existence of magnetic monopoles are listed below; a more comprehensive review is given in Ref. [19].

Dirac monopole In Dirac's formulation [1,20], magnetic monopoles are assumed to be point-like particles with quantum mechanical conditions leading to Equation (1), establishing the discrete nature of their magnetic charge. In spite of monopoles formally symmetrising the Maxwell's equations, a numerical asymmetry emerges in the DQC: the minimum value of the magnetic charge is much larger than the smallest electric charge. Indeed, a magnetic monopole with a single Dirac charge $g_{\mathrm{D}}$ has an equivalent electric charge of $137 \beta e / 2$. Hence, for a relativistic monopole, the energy loss is around $68.5^{2} \simeq 4700$ times that of a minimum-ionising particle.

Monopoles in GUTs Since the Grand Unified Theory (GUT) of strong and electroweak interactions predicted the existence of magnetic monopoles [21,22], searches for magnetic 
monopoles, in particular of cosmic origin, have been intensified substantially. In 1974, 't Hooft [2] and Polyakov [3] showed that a unified gauge theory where electromagnetism is embedded in a semi-simple gauge group, such as $S U(2)$, would necessitate the existence of the monopole as a soliton with spontaneous symmetry breaking. GUT monopoles are too massive to be produced at any future accelerator, having a mass of $\mathcal{O}\left(10^{15} \mathrm{GeV}\right)$ [23].

Electroweak monopole Cho and Maison postulated the electroweak monopole $[17,18,24,25]$ as a generalisation of the Dirac monopole, representing a hybrid of Dirac and 't Hooft-Polyakov monopoles that carries magnetic charge twice that of the Dirac monopole. The latter is due to the quotient group $S U(2) \otimes U_{Y}(1) / U_{\mathrm{em}}(1)$, where $U_{\mathrm{em}}(1)$, which is the (unbroken) group of electromagnetism instead of, e.g., the $S U(2)$ group in the Georgi-Glashow model. Recent estimates of the electroweak monopole mass [26] indicate that it is possibly accessible at the LHC.

Global monopoles They have been proposed [27] as space-time (cosmological) defects allowing for the spontaneous breaking of internal global $S O(3)$ symmetries in non-gauged Georgi-Glashow models. These monopoles carry no magnetic charge, yet gravitational effects away from their centre are significant, leading to a deficit angle in the (non-Minkowski) space-time. Such an effect may modify the forward scattering amplitude of Standard Model (SM) background particles, creating ring-like angular regions with very large scattering amplitude [28,29]. Such peculiar scattering patterns of ordinary SM particles may indicate indirectly the presence of a neutral global monopole in collider detectors, where they may be pair-produced [27,30]. Moreover, a variant of the global monopole model, including axion fields and a real electromagnetic field, coupling only gravitationally to the scalar $S O(3)$ symmetry breaking sector, has been proposed [31-33], resulting in axions capable of inducing electromagnetic monopole solutions with a real magnetic charge.

Monopolium The lack of experimental confirmation of monopoles in Dirac's proposal $[1,20,34]$ may be attributed to monopoles not being seen freely because they form a bound state called monopolium [35-38], confined by strong magnetic forces. Monopolium is a neutral state, hence it is difficult to detect directly at a collider detector; however, its decay into photons would give a rather clear signal in the ATLAS, CMS and CMS-TOTEM Precision Proton Spectrometer (CT-PPS) detectors [39-43].

\section{Searches for Cosmic Monopoles}

Magnetic monopoles of cosmic origin are hypothesised to have been formed shortly after the Big Bang as topological defects arising when the Universe expanded and cooled. The existing galactic magnetic field $B \simeq 3 \mu \mathrm{G}$ would accelerate such monopoles, thus draining energy from the magnetic field, so its dissipation should not exceed its regeneration, should the galactic field be sustained. This requirement implies that an upper flux limit should be respected, the so-called Parker bound [44]

$$
\Phi \lesssim 10^{-15} \mathrm{~cm}^{-2} \mathrm{~s}^{-1} \mathrm{sr}^{-1} .
$$

Blas Cabrera and collaborators at Stanford University prepared an experiment with a four-turn, 5-cm-diameter loop, vertically oriented, connected to the superconducting input coil of a superconducting quantum interference device (SQUID) [45]. They observed a single candidate event during the 151 days of running of this experiment on 14 February 1982. If this candidate event is considered to be spurious, an upper limit of $6.1 \times 10^{-10} \mathrm{~cm}^{-2} \mathrm{~s}^{-1} \mathrm{sr}^{-1}$ is derived, which is much larger than the Parker bound. Despite further improvements of the experimental setup to suppress possible background sources, this observation was not replicated. The best bound given by an induction detector on cosmic monopoles was obtained later by the same group: a 90\% confidence limit (CL) on the monopole flux of $7.2 \times 10^{-10} \mathrm{~cm}^{-2} \mathrm{~s}^{-1} \mathrm{sr}^{-1}[46]$.

Other direct cosmic searches have been performed on underground, surface and balloon-borne experiments targeting GUT monopoles in a mass range of $100 \div 10^{4} \mathrm{TeV}$ with a velocity spanning 
$\beta \sim 10^{-4} \div 1$. Up to now, there is no experimental evidence for cosmic magnetic monopoles-only bounds on their flux as a function of mass and velocity, summarised in Figure 7 of Ref. [47].

MACRO [48] was a large underground detector situated in the Gran Sasso laboratory. It has provided the best limits so far for GUT monopoles with a sensitivity that largely covers the velocity range, mostly thanks to the redundancy and complementarity of the various detector components it was comprising: liquid scintillation counters; limited streamer tubes; and nuclear track detectors (NTDs). However, due to its underground location, it was not sensitive to lower-energy monopoles, which are blocked by the Earth. MACRO set an upper limit on the monopole flux well below the Parker bound in almost all the $\beta$ range for GUT monopoles [49]. Limits were also set by an experiment at the Ohya stone quarries in Japan [50], featuring an array of CR-39 NTDs, while another experiment at Baksan [51] in Russia used liquid scintillation counters. Soudan 2 [52], in the United States, was a large, fine-grained tracking calorimeter composed of long drift tubes, and another tracking calorimeter, Kolar Gold Fields (KGF) [53], was deployed in India.

The SLIM detector, on the other hand, installed at high altitude in Bolivia at an altitute of $5400 \mathrm{~m}$, probed a region for intermediate-mass monopoles $\left(10^{5} \lesssim M \lesssim 10^{12} \mathrm{GeV}\right)$, well below the GUT scale, which do not have enough energy to penetrate the entire atmosphere. The SLIM NTDs array covered an area $>400 \mathrm{~m}^{2}$ that, after four years of exposure, showed no signal of magnetic monopoles and set limits on cosmic monopole flux [54].

Relativistic monopoles can be sought by the emittance of Cherenkov radiation, when traveling through a homogeneous and transparent medium such as water or ice, which can be detected by arrays or strings of photomultiplier tubes. Neutrino telescopes such as Baikal [55], AMANDA [56], ANTARES $[57,58]$ and IceCube $[59,60]$ are sensitive to visible Cherenkov light emitted by a monopole with $\beta>0.75$ (direct Cherenkov). Additional light is produced by Cherenkov radiation from $\delta$-ray electrons along the monopole path for velocities down to $\beta=0.625$ (indirect Cherenkov). Furthermore luminescence may be induced by molecular excitation of the medium for monopole velocities of $\beta>0.01$. Results for relativistic monopoles from MACRO [49], ANTARES [58] and IceCube [60,61] are depicted in Figure 1 [62]. As is the case for neutrinos, a large background from cosmic muons inhibits searches for down-going candidates; up-going monopoles having traversed the Earth before reaching the detector are probed instead.

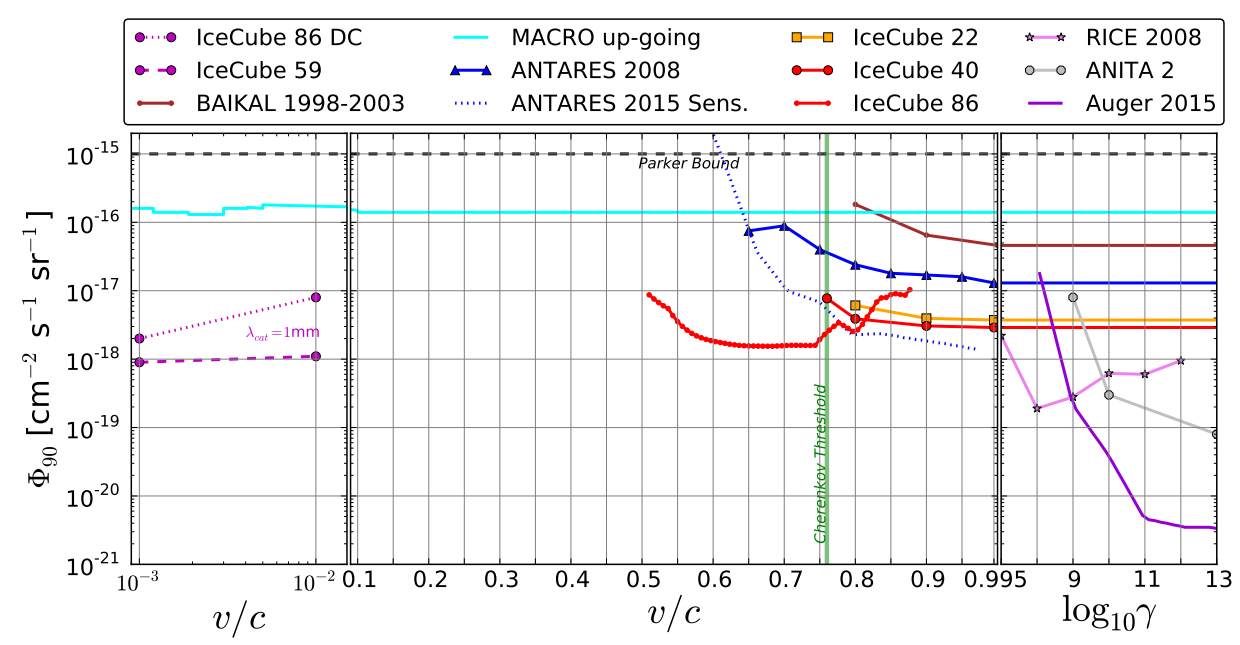

Figure 1. 90\% CL upper limits versus velocity $\beta$ for a flux of very energetic cosmic GUT monopoles with magnetic charge of $g=g_{\mathrm{D}}$. The IceCube $86 \mathrm{DC}$ nucleon-decay analysis is based on the IceCube DeepCore [61]. Results from MACRO [49], ANTARES [57], RICE [63], BAIKAL [55], ANITA [64], and Auger [65] are superimposed on IceCube limits from direct (IceCube 40) [59] and indirect (IceCube 86) Cherenkov light [60]—from Ref. [62]. 
The flux of ultra-relativistic monopoles has been constrained by the Pierre Auger Observatory [65], which was sensitive to monopoles with Lorentz factor values $\gamma \sim 10^{9} \div 10^{12}$. Two other experiments exploited the radio-wave pulses from the interactions of a primary particle with ice to search for monopoles. The Radio Ice Cherenkov Experiment (RICE), consisting of radio antennas buried in the Antarctic ice, set a flux upper limit of $10^{-18} \mathrm{~cm}^{-2} \mathrm{~s}^{-1} \mathrm{sr}^{-1}$ at $95 \% \mathrm{CL}$ for intermediate-mass monopoles with $10^{7}<\gamma<10^{12}$ and a total energy of $10^{16} \mathrm{GeV}$ [63]. The ANITA-II balloon-borne radio interferometer, on the other hand, set a $90 \%$-CL flux upper limit on the order of $10^{-19} \mathrm{~cm}^{-2} \mathrm{~s}^{-1} \mathrm{sr}^{-1}$ for a Lorentz factor $\gamma>10^{10}$ at a total energy of $10^{16} \mathrm{GeV}$ [64].

\section{Searches in Collider Experiments}

Present and proposed future accelerators feature a centre-of-mass energy of $\mathcal{O}(10 \mathrm{TeV})$, thus it is practically impossible to search for GUT monopoles in these machines. Nevertheless, searches have been carried out to detect direct or indirect signals of lower-mass monopoles. Searches have been performed at hadron-hadron, electron-positron and lepton-hadron experiments, mostly directly using scintillation counters, gas chambers and NTDs, taking advantage of the monopole high ionisation power $[19,66,67]$. Other analyses focus on exposed material for trapped monopoles or peculiar magnetic-charge trajectories. In addition, virtual-monopole processes enhancing production rates of certain final states have also been considered as indirect probes for monopoles.

\subsection{Past Searches}

Collider experiments typically express their results in terms of upper limits on a production cross section versus the monopole mass. To calculate these limits, an ansatz is used to model the kinematics of monopole-antimonopole pair production processes since perturbative field theory cannot be used to calculate the rate and kinematic properties of produced monopoles [68]. Limits therefore suffer from a degree of model-dependence, implying that a comparison between the results of different experiments can be problematic, in particular when this concerns excluded mass regions. This situation may be resolved if thermal production in heavy-ion collisions-that does not rely on perturbation theory-is considered [69,70]. Another recent proposal involves, for the cases of spin- $1 / 2$ and spin- 1 monopoles, the introduction of a magnetic-moment term, which, together with the velocity-dependent coupling, allows for a perturbative treatment of the cross-section calculation [71-73]. Specifically, this may be possible if large values of the magneticmoment parameter and slow-moving monopoles are considered.

At the Tevatron, the CDF [74] and E882 [75] experiments of Tevatron have performed searches for monopoles. The CDF experiment used a dedicated time-of-flight system while the E882 experiment employed the induction technique to search for stopped monopoles in discarded material exposed to $p \bar{p}$ collisions. Earlier searches at the Tevatron, such as at the D0 collision point [76], used NTDs and were based on comparatively modest amounts of integrated luminosity. Lower energy hadron-hadron experiments have employed a variety of search techniques including plastic track detectors at the CERN Spp̄S [77] and searches for trapped monopoles in a 300-GeV-proton beam dump [78].

The only LEP-2 search was made by OPAL [79] which quoted cross section limits for the production of monopoles with masses up to around $103 \mathrm{GeV}$. At LEP-1, searches were made with NTDs deployed around an interaction region, thus allowing high charges to be probed for masses up to $\sim 45 \mathrm{GeV}$. Specifically, the L6-MODAL experiment [80] set limits for monopoles with charges in the range $0.9 \div 3.6 g_{\mathrm{D}}$, whilst an earlier search by the MODAL experiment was sensitive to monopoles with charges as low as $0.1 g_{\mathrm{D}}$ [81]. The deployment of NTDs around the beam interaction point was also used at earlier $e^{+} e^{-}$colliders such as KEK by TRISTAN [82] and PETRA [83] at DESY. Searches at $e^{+} e^{-}$facilities have also been made for particles following non-helical trajectories with the CLEO [84] and TASSO [85] detectors. 


\subsection{Searches for Monopoles in ATLAS}

At the ATLAS experiment [86], searches for magnetic monopoles have been performed on 7-TeV [87] and on 8-TeV [88] data using the transition radiation tracker (TRT) [89,90] sensitivity to high-ionisation signals. The 8-TeV analysis relies on a dedicated trigger for highly-ionising particles, which makes use of the fraction of TRT hits passing a predefined high threshold, $f_{\mathrm{HT}}$. The discriminating particle characteristics used by this search are the energy dispersion in the electromagnetic calorimeter, $w$, and the $f_{\mathrm{HT}}$. The energy dispersion measures the fraction of the cluster energy contained in the most energetic cells of a cluster in each of the layers of the electromagnetic calorimeters.

Since no excess of data events was observed, the search is interpreted assuming the Drell-Yan (DY) production process with modified electromagnetic couplings as seen in Figure 2(a) [88]. The analysis is sensitive to magnetic charges of $0.5 g_{\mathrm{D}} \leq|g| \leq 1.5 g_{\mathrm{D}}$ and sets limits for spin- 0 and spin-1/2 monopoles. The search excludes monopoles with a magnetic charge of $1 g_{\mathrm{D}}$ up to masses of $1340 \mathrm{GeV}$ for a spin-1/2 hypothesis of the particle. In addition, a model independent cross section upper limit of $0.5 \mathrm{fb}$ is set in fiducial regions where the selection efficiency is almost constant. This analysis has been updated recently by ATLAS with $13-\mathrm{TeV} p p$ collision data [91].

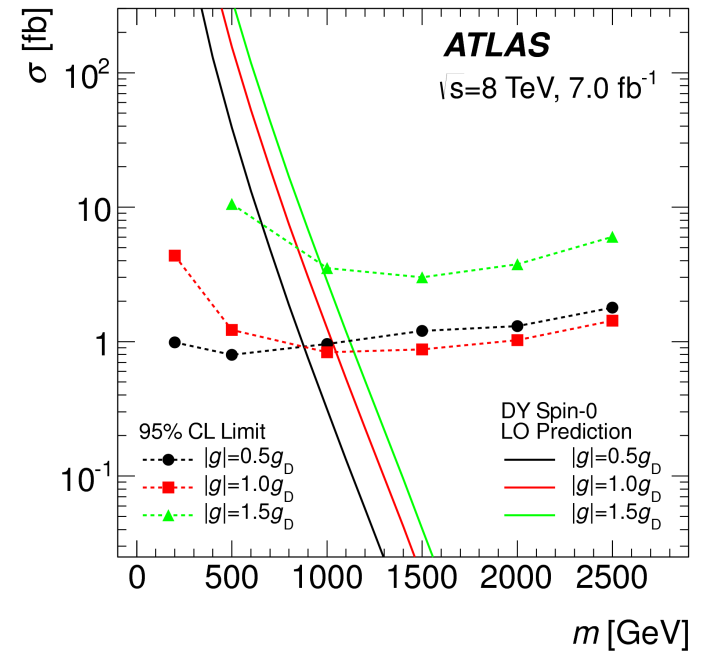

(a) ATLAS results.

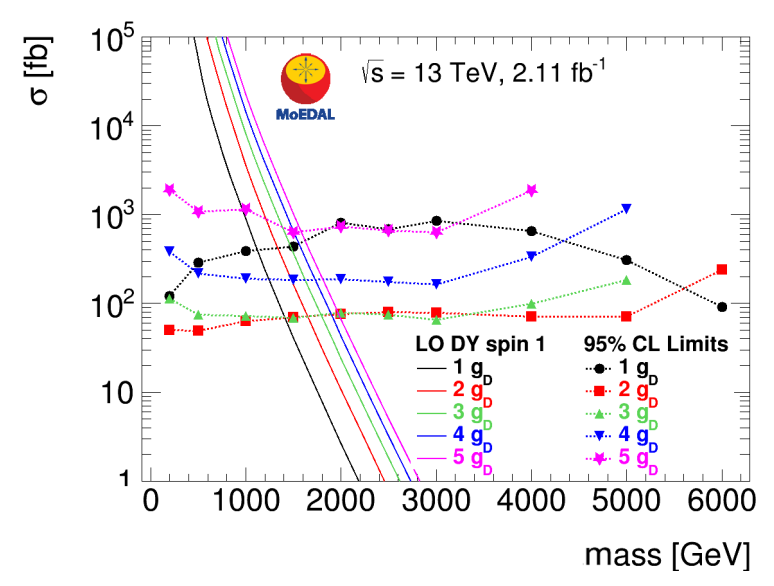

(b) MoEDAL results.

Figure 2. (a) 95\% cross-section upper limits (dashed lines) and theoretical cross sections (solid lines) for magnetic monopoles with spin 0 and various magnetic charges-from Ref. [88]; (b) cross-section upper limits at $95 \%$ confidence level for DY monopole production as a function of mass for spin-1 monopoles. The various line colours correspond to different monopole charges. The solid lines represent DY cross-section calculations at leading order. From Ref. [92].

\subsection{MoEDAL Experiment}

MoEDAL (Monopole and Exotics Detector at the LHC) [93-95] is designed to search for manifestations of new physics through highly ionising particles in a manner complementary to ATLAS and CMS [96]. The main motivation for the MoEDAL experiment is to pursue the quest for magnetic monopoles at LHC energies [97]. Nonetheless, the detector is also designed to search for any massive, long-lived, slow-moving particles $[98,99]$ with single or multiple electric charges arising in many scenarios of physics beyond the SM [97].

The MoEDAL detector $[93,97]$ is deployed around the intersection region at Point 8 of the LHC in the LHCb experiment Vertex Locator cavern. It is a unique and largely passive LHC detector comprised of three main sub-detectors:

Nuclear track detectors The main sub-detector system is made of a large array of CR-39, Makrofol ${ }^{\circledR}$ and Lexan ${ }^{\mathrm{TM}}$ NTD stacks surrounding the intersection area. The passage of an 
HI particle through the plastic detector is marked by an invisible damage zone along the trajectory. The damage zone is revealed as a cone-shaped etch-pit when the plastic detector is chemically etched. Then, the sheets of plastics are scanned looking for aligned etch pits in multiple sheets. The MoEDAL NTDs have a (low) threshold of $z / \beta \sim 5$, where $z$ is the charge and $\beta=v / c$ the velocity of the incident particle. Another type of NTD installed is the Very High Charge Catcher $(z / \beta \sim 50)$, consisting of two flexible low-mass stacks of Makrofol ${ }^{\circledR}$. It is the only NTD (partly) covering the forward region, being deployed in the $\mathrm{LHCb}$ acceptance between RICH1 and the Trigger Tracker.

Magnetic trappers A unique feature of the MoEDAL detector is the use of paramagnetic magnetic monopole trappers (MMTs) to capture magnetically-charged HI particles. The aluminium absorbers of MMTs are subject to an analysis looking for magnetically charged particles at a remote SQUID magnetometer facility [100].

TimePix radiation monitors The only non-passive MoEDAL sub-detector is an array of TimePix pixel devices distributed throughout the MoEDAL cavern, forming a real-time radiation monitoring system of beam-related backgrounds. The operation in time-over-threshold mode helps differentiating between various particles species from mixed radiation fields and measuring their energy deposition.

The high ionisation of slow-moving magnetic monopoles implies quite characteristic trajectories when such particles interact with the MoEDAL NTDs, which can be revealed during the etching process $[93,97]$. In addition, the high magnetic charge of a monopole (which is expected to be at least one Dirac charge $g_{\mathrm{D}}=68.5$ e (cf. (1)) implies a strong magnetic dipole moment, which in turn may result in a strong binding of the monopole with the aluminum nuclei of the MoEDAL MMTs. In such a case, the presence of a monopole trapped in an aluminium bar of an MMT would be detected through the existence of a persistent current, defined as the difference between the currents in the SQUID of a magnetometer before and after the passage of the bar through the sensing coil.

In the context of the MMT exposure during 2015-2016 of Run 2, no magnetic charge exceeding $0.5 g_{D}$ was detected in any of the exposed samples when passed through the ETH Zurich SQUID facility, allowing limits to be placed on monopole production. Model-independent cross-section limits have been obtained in fiducial regions of monopole energy and direction for $1 g_{\mathrm{D}} \leq|g| \leq 6 g_{\mathrm{D}}$ with the 8-TeV analysis [101]. The latest model-dependent cross-section limits are obtained for DY pair production of spin-1, spin-1/2 and spin-0 monopoles for $1 g_{\mathrm{D}} \leq|g| \leq 5 g_{\mathrm{D}}$ at $13 \mathrm{TeV}$ [92], as shown in Figure $2 \mathrm{~b}$ for spin-1. This analysis extended previously obtained bounds [102] and added new interpretations for a $\beta$-dependent coupling and for the first time for spin-1 monopoles. Monopole production via photon fusion has also been recently considered in MoEDAL monopole search analyses [103] following related phenomenological studies [71].

\section{Conclusions and Outlook}

The existence of magnetic monopoles should be confirmed experimentally and would modify our understanding of electrodynamics, rendering the Maxwell equation fully symmetric. The Dirac quantisation condition is a beautiful consequence of the existence of monopoles and therefore they represent an extremely appealing physical scenario. Various searches have been carried out utilising diverse detection techniques in both observational facilities and experiments in colliders.

Neutrino telescopes, such as ANTARES and IceCube currently, and KM3NeT and PINGU in the future, pursue the search for GUT monopoles in the cosmic front.

The CERN LHC, being the most powerful collider to-date, is the ideal machine to produce magnetic monopoles, if they exist. In particular, the MoEDAL experiment is specialising in the detection of magnetic monopoles and other highly ionising particles. MoEDAL has set the stringent bounds on high magnetic charges [103] and together with other LHC experiments, such as ATLAS [91] and possibly CMS, are going to continue probing the existence of light monopoles. Moreover, volumes exposed to LHC collisions, such as the Run-1 CMS beam pipe [104], will be 
scrutinised by SQUID for the presence of trapped monopoles. Another possibility to probe magnetic monopoles is given by their bound state, the monopolium, through their multiphoton final states [39-42] that can be tested in ATLAS and CMS.

Funding: This research was funded by the Generalitat Valenciana (GV) through specific MoEDAL-supporting agreements and the GV Excellence Project PROMETEO-II/2017/033, by the Spanish MICIU under the projects FPA2015-65652-C4-1-R and PGC2018-094856-B-I00, by the Severo Ochoa Excellence Centre Project SEV-2014-0398 and by a 2017 Leonardo Grant for Researchers and Cultural Creators, BBVA Foundation.

Conflicts of Interest: The author declares no conflict of interest. The funding sponsors had no role in the design of the study; in the collection, analyses, or interpretation of data; in the writing of the manuscript, or in the decision to publish the results.

\section{Abbreviations}

The following abbreviations are used in this manuscript:

$\begin{array}{ll}\text { AMANDA } & \text { Antarctic Muon And Neutrino Detector Array } \\ \text { ANITA } & \text { Antarctic Impulse Transient Antenna } \\ \text { ANTARES } & \text { Astronomy with a Neutrino Telescope and Abyss environmental RESearch } \\ \text { ATLAS } & \text { A Toroidal LHC ApparatuS } \\ \text { CDF } & \text { Collider Detector at Fermilab } \\ \text { CL } & \text { Confidence Level } \\ \text { CT-PPS } & \text { CMS-TOTEM Precision Proton Spectrometer } \\ \text { DQC } & \text { Dirac Quantisation Condition } \\ \text { DY } & \text { Drell-Yan } \\ \text { GUT } & \text { Grand Unified Theory } \\ \text { HI } & \text { Highly Ionising } \\ \text { KGF } & \text { Kolar Gold Fields } \\ \text { LEP } & \text { Large Electron-Positron Collider } \\ \text { LHC } & \text { Large Hadron Collider } \\ \text { MACRO } & \text { Monopole, Astrophysics and Cosmic Ray Observatory } \\ \text { MMT } & \text { Magnetic Monopole Trapper } \\ \text { MODAL } & \text { MOnopole Detector At LEP } \\ \text { MoEDAL } & \text { Monopole and Exotics Detector At the LHC } \\ \text { NTD } & \text { Nuclear Track Detector } \\ \text { PINGU } & \text { Precision IceCube Next, Generation Upgrade } \\ \text { RICE } & \text { Radio Ice Cherenkov Experiment } \\ \text { SLIM } & \text { Search for LIght magnetic Monopoles } \\ \text { SM } & \text { Standard Model } \\ \text { SQUID } & \text { Superconducting QUantum Interference Device } \\ \text { TRT } & \text { Transition Radiation Tracker } \\ \text { VHCC } & \text { Very High Charge Catcher } \\ & \end{array}$

\section{References}

1. Dirac, P.A.M. Quantized Singularities in the Electromagnetic Field. Proc. R. Soc. Lond. A 1931, 133, 60-72, doi:10.1098/rspa.1931.0130.

2. Hooft, G. Magnetic Monopoles in Unified Gauge Theories. Nucl. Phys. B 1974, 79, 276-284, doi:10.1016/0550-3213(74)90486-6.

3. Polyakov, A.M. Particle Spectrum in the Quantum Field Theory. JETP Lett. 1974, 20, 194

4. Julia, B.; Zee, A. Poles with Both Magnetic and Electric Charges in Nonabelian Gauge Theory. Phys. Rev. D 1975, 11, 2227-2232, doi:10.1103/PhysRevD.11.2227.

5. Nambu, Y. String-Like Configurations in the Weinberg-Salam Theory. Nucl. Phys. B 1977, 130, 505-515, doi:10.1016/0550-3213(77)90252-8.

6. Witten, E. Dyons of Charge $e \theta / 2 \pi$. Phys. Lett. 1979, 86, 283-287, doi:10.1016/0370-2693(79)90838-4. 
7. Lazarides, G.; Magg, M.; Shafi, Q. Phase Transitions and Magnetic Monopoles in SO(10). Phys. Lett. 1980, 97, 87-92, doi:10.1016/0370-2693(80)90553-5.

8. Sorkin, R.D. Kaluza-Klein Monopole. Phys. Rev. Lett. 1983, 51, 87-90, doi:10.1103/PhysRevLett.51.87.

9. Gross, D.J.; Perry, M.J. Magnetic Monopoles in Kaluza-Klein Theories. Nucl. Phys. B 1983, 226, 29-48, doi:10.1016/0550-3213(83)90462-5.

10. Schwinger, J.S. A Magnetic model of matter. Science 1969, 165, 757-761, doi:10.1126/science.165.3895.757.

11. Preskill, J. Magnetic Monopoles. Ann. Rev. Nucl. Part. Sci. 1984, 34, 461-530, doi:10.1146/annurev.ns.34. 120184.002333.

12. Achucarro, A.; Vachaspati, T. Semilocal and electroweak strings. Phys. Rep. 2000, 327, 347-426, doi:10.1016/S0370-1573(99)00103-9.

13. Kephart, T.W.; Lee, C.A.; Shafi, Q. Family unification, exotic states and light magnetic monopoles. JHEP 2007, 0701, 088, doi:10.1088/1126-6708/2007/01/088.

14. Pak, D.G.; Zhang, P.M.; Zou, L.P. On finite energy monopole solutions in Weinberg-Salam model. Int. J. Mod. Phys. A 2015, 30, 1550164, doi:10.1142/S0217751X1550164X.

15. Rajantie, A. Mass of a quantum 't Hooft-Polyakov monopole. JHEP 2006, 0601, 088, doi:10.1088/1126-6708/2006/01/088.

16. Kephart, T.W.; Leontaris, G.K.; Shafi, Q. Magnetic Monopoles and Free Fractionally Charged States at Accelerators and in Cosmic Rays. JHEP 2017, 1710, 176, doi:10.1007/JHEP10(2017)176.

17. Cho, Y.M.; Maison, D. Monopoles in Weinberg-Salam model. Phys. Lett. B 1997, 391, 360-365, doi:10.1016/S0370-2693(96)01492-X.

18. Bae, W.S.; Cho, Y.M. Finite energy electroweak dyon. J. Korean Phys. Soc. 2005, 46, 791-804.

19. Mavromatos, N.E.; Mitsou, V.A. Magnetic monopoles revisited: Models and searches at colliders and in the Cosmos. Mod. Phys. Lett. A, in preparation.

20. Dirac, P.A.M. The Theory of magnetic poles. Phys. Rev. 1948, 74, 817-830, doi:10.1103/PhysRev.74.817.

21. Rajantie, A. Introduction to Magnetic Monopoles. Contemp. Phys. 2012, 53, 195-211, doi:10.1080/00107514.2012.685693.

22. Rajantie, A. The search for magnetic monopoles. Phys. Today 2016, 69, 40-46, doi:10.1063/PT.3.3328.

23. Vento, V.; Mantovani, V.S. On the magnetic monopole mass. arXiv 2013, arXiv:1306.4213.

24. Cho, Y.M.; Kimm, K.; Yoon, J.H. Mass of the Electroweak Monopole. Mod. Phys. Lett. A 2016, 31, 1650053, doi:10.1142/S021773231650053X.

25. Cho, Y.M.; Kimm, K.; Yoon, J.H. Gravitationally Coupled Electroweak Monopole. Phys. Lett. B 2016, 761, 203-206, doi:10.1016/j.physletb.2016.08.033.

26. Ellis, J.; Mavromatos, N.E.; You, T. The Price of an Electroweak Monopole. Phys. Lett. B 2016, 756, 29-35, doi:10.1016/j.physletb.2016.02.048.

27. Barriola, M.; Vilenkin, A. Gravitational Field of a Global Monopole. Phys. Rev. Lett. 1989, 63, 341-343, doi:10.1103/PhysRevLett.63.341.

28. Mazur, P.O.; Papavassiliou, J. Gravitational scattering on a global monopole. Phys. Rev. D 1991, 44, 1317-1320, doi:10.1103/PhysRevD.44.1317.

29. Mavromatos, N.E.; Papavassiliou, J. Singular lensing from the scattering on special space-time defects. Eur. Phys. J. C 2018, 78, 68, doi:10.1140/epjc/s10052-018-5542-5.

30. Drukier, A.K.; Nussinov, S. Monopole Pair Creation in Energetic Collisions: Is It Possible? Phys. Rev. Lett. 1982, 49, 102-105, doi:10.1103/PhysRevLett.49.102.

31. Mavromatos, N.E.; Sarkar, S. Magnetic monopoles from global monopoles in the presence of a Kalb-Ramond field. Phys. Rev. D 2017, 95, 104025, doi:10.1103/PhysRevD.95.104025.

32. Mavromatos, N.E.; Sarkar, S. Regularised Kalb-Ramond Magnetic Monopole with Finite Energy. Phys. Rev. D 2018, 97, 125010, doi:10.1103/PhysRevD.97.125010.

33. Sarkar, S.; Mavromatos, N.E. Finite-Energy Dressed String-Inspired Dirac-Like Monopoles. Universe 2019, 5, 8, doi:10.3390/universe5010008.

34. Zeldovich, Y.B.; Khlopov, M.Y. On the Concentration of Relic Magnetic Monopoles in the Universe. Phys. Lett. 1978, 79, 239-241, doi:10.1016/0370-2693(78)90232-0.

35. Hill, C.T. Monopolonium. Nucl. Phys. B 1983, 224, 469-490, doi:10.1016/0550-3213(83)90386-3.

36. Dubrovich, V.K. Association of magnetic monopoles and antimonopoles in the early universe. Grav. Cosmol. Suppl. 2002, 8, 122-125. 
37. Epele, L.N.; Fanchiotti, H.; Garcia, Canal, C.A.; Vento, V. Monopolium: The Key to monopoles. Eur. Phys. J. C 2008, 56, 87-95, doi:10.1140/epjc/s10052-008-0628-0.

38. Epele, L.N.; Fanchiotti, H.; Garcia, Canal, C.A.; Vento, V. Monopolium production from photon fusion at the Large Hadron Collider. Eur. Phys. J. C 2009, 62, 587-592, doi:10.1140/epjc/s10052-009-1069-0.

39. Epele, L.N.; Fanchiotti, H.; Garcia, Canal, C.A.; Mitsou, V.A.; Vento, V. Looking for magnetic monopoles at LHC with diphoton events. Eur. Phys. J. Plus 2012, 127, 60, doi:10.1140/epjp/i2012-12060-8.

40. Epele, L.N.; Fanchiotti, H.; Garcia, Canal, C.A.; Mitsou, V.A.; Vento, V. Can the $750 \mathrm{GeV}$ enhancement be a signal of light magnetic monopoles? arXiv 2016, arXiv:1607.05592.

41. Fanchiotti, H.; Garcia, Canal, C.A.; Vento, V. Multiphoton annihilation of monopolium. Int. J. Mod. Phys. A 2017, 32, 1750202, doi:10.1142/S0217751X17502025.

42. Vento, V. Ions, Protons, and Photons as Signatures of Monopoles. Universe 2018, 4, 117, doi:10.3390/universe4110117.

43. Baldenegro, C.; Fichet, S.; von Gersdorff, G.; Royon, C. Searching for axion-like particles with proton tagging at the LHC. JHEP 2018, 1806, 131, doi:10.1007/JHEP06(2018)131.

44. Turner, M.S.; Parker, E.N.; Bogdan, T.J. Magnetic Monopoles and the Survival of Galactic Magnetic Fields. Phys. Rev. D 1982, 26, 1296-1305, doi:10.1103/PhysRevD.26.1296.

45. Cabrera, B. First Results from a Superconductive Detector for Moving Magnetic Monopoles. Phys. Rev. Lett. 1982, 48, 1378-1380, doi:10.1103/PhysRevLett.48.1378.

46. Huber, M.E.; Cabrera, B.; Taber, M.A.; Gardner, R.D. Limit on the flux of cosmic ray magnetic monopoles from operation of an eight loop superconducting detector. Phys. Rev. Lett. 1990, 64, 835-838, doi:10.1103/PhysRevLett.64.835.

47. Patrizii, L.; Spurio, M. Status of Searches for Magnetic Monopoles. Ann. Rev. Nucl. Part. Sci. 2015, 65, 279-302, doi:10.1146/annurev-nucl-102014-022137.

48. Ambrosio, M.; Antolini, R.; Assiro, R.; Auriemma, G.; Bakari, D.; Baldini, A.; Barbarino, G.C.; Barbarito, E.; Barish, B.C.; Battistoni, G.; et al. The MACRO detector at Gran Sasso. Nucl. Instrum. Meth. A 2002, 486, 663-707, doi:10.1016/S0168-9002(01)02169-6.

49. MACRO Collaboration. Final results of magnetic monopole searches with the MACRO experiment. Eur. Phys. J. C 2002, 25, 511-522, doi:10.1140/epjc/s2002-01046-9.

50. Orito, S.; Ichinose, H.; Nakamura, S.; Kuwahara, K.; Doke, T.; Ogura, K.; Tawara, H.; Imori, M.; Yamamoto, K.; Yamakawa, H.; et al. Search for supermassive relics with 2000- $\mathrm{m}^{2}$ array of plastic track detector. Phys. Rev. Lett. 1991, 66, 1951-1954, doi:10.1103/PhysRevLett.66.1951.

51. Novoseltsev, Y.F.; Boliev, M.M.; Butkevich, A.V.; Mikheev, S.P. Petkov, V.B. A search for massive magnetic monopoles at the Baksan Underground Scintillation Telescope (BUST). Nucl. Phys. Proc. Suppl. 2006, 151, 337-340, doi:10.1016/j.nuclphysbps.2005.07.048.

52. Thron, J.L.; Allison, W.W.M.; Alner, G.J.; Ambats, I.; Ayres, D.S.; Balka, L.J.; Barr, G.D.; Barrett, W.L.; Benjamin, D.; Border, P.M.; et al. Search for magnetic monopoles with the Soudan 2 detector. Phys. Rev. D 1992, 46, 4846-4851, doi:10.1103/PhysRevD.46.4846.

53. Krishnaswamy, M.R.; Menon, M.G.K.; Mondal, N.K.; Narasimham, V.S.; Sreekantan, B.V.; Hayashi, Y.; Ito, N.; Kawakami, S.; Miyake, S. Limits On The Flux Of Monopoles From The Kolar Gold Mine Experiments. Phys. Lett. 1984, 142, 99-102, doi:10.1016/0370-2693(84)91143-2.

54. Balestra, S.; Cecchini, S.; Cozzi, M.; Errico, M.; Fabbri, F.; Giacomelli, G.; Giacomelli, R.; Giorgini, M.; Kumar, A.; Manzoor, S; et al. Magnetic monopole search at high altitude with the SLIM experiment. Eur. Phys. J. C 2008, 55, 57-63, doi:10.1140/epjc/s10052-008-0597-3.

55. Aynutdinov, V.; Avrorin, A.; Balkanov, V.; Belolaptikov, I.; Budnev, N.; Danilchenko, I.; Domogatsky, G.; Doroshenko, A.; Dyachok, A.; Dzhilkibaev, Z.-A.; et al. Search for relativistic magnetic monopoles with the Baikal Neutrino Telescope. Astropart. Phys. 2008, 29, 366-372, doi:10.1016/j.astropartphys.2008.03.006.

56. Abbasi, R.; Abdou, Y.; Abu-Zayyad, T.; Adams, J.; Aguilar, J.A.; Ahlers, M.; Andeen, K.; Auffenberg, J.; Bai, X.; Baker, M.; et al. Search for relativistic magnetic monopoleswith the AMANDA-II neutrino telescope. Eur. Phys. J. C 2010, 69, 361-378, doi:10.1140/epjc/s10052-010-1411-6.

57. ANTARES Collaboration. Search for Relativistic Magnetic Monopoles with the ANTARES Neutrino Telescope. Astropart. Phys. 2012, 35, 634-640, doi:10.1016/j.astropartphys.2012.02.007.

58. ANTARES Collaboration. Search for relativistic magnetic monopoles with five years of the ANTARES detector data. JHEP 2017, 1707, 054, doi:10.1007/JHEP07(2017)054. 
59. IceCube Collaboration. Search for relativistic magnetic monopoles with IceCube. Phys. Rev. D 2013, 87, 022001, doi:10.1103/PhysRevD.87.022001.

60. IceCube Collaboration. Searches for relativistic magnetic monopoles in IceCube. Eur. Phys. J. C 2016, 76, 133, doi:10.1140/epjc/s10052-016-3953-8.

61. IceCube Collaboration. Search for non-relativistic magnetic monopoles with IceCube. Eur. Phys. J. C 2014, 74, 2938, doi:10.1140/epjc/s10052-014-2938-8.

62. IceCube Collaboration. Luminescence of water or ice as a new detection method for magnetic monopoles. EPJ Web Conf. 2017, 164, 07019, doi:10.1051/epjconf/201716407019.

63. Hogan, D.P.; Besson, D.Z.; Ralston, J.P.; Kravchenko, I.; Seckel, D. Relativistic Magnetic Monopole Flux Constraints from RICE. Phys. Rev. D 2008, 78, 075031, doi:10.1103/PhysRevD.78.075031.

64. ANITA-II Collaboration. Ultrarelativistic magnetic monopole search with the ANITA-II balloon-borne radio interferometer. Phys. Rev. D 2011, 83, 023513, doi:10.1103/PhysRevD.83.023513.

65. Pierre Auger Collaboration. Search for ultrarelativistic magnetic monopoles with the Pierre Auger observatory. Phys. Rev. D 2016, 94, 082002, doi:10.1103/PhysRevD.94.082002.

66. Lee, L.; Ohm, C.; Soffer, A.; Yu, T.T. Collider Searches for Long-Lived Particles Beyond the Standard Model. Prog. Part. Nucl. Phys. 2019, 106, 210, doi:10.1016/j.ppnp.2019.02.006.

67. Alimena, J.; Beacham, J.; Borsato, M.; Cheng, Y.; Vidal, X.C.; Cottin, G.; Roeck, A.D.; Desai, N.; Curtin, D.; Evans, J.A.; et al. Searching for Long-Lived Particles beyond the Standard Model at the Large Hadron Collider. arXiv 2019, arXiv:1903.04497.

68. Particle Data Group. Review of Particle Physics. Chin. Phys. C 2016, 40, 100001, doi:10.1088/1674-1137/40/10/100001.

69. Gould, O.; Rajantie, A. Magnetic monopole mass bounds from heavy ion collisions and neutron stars. Phys. Rev. Lett. 2017, 119, 241601, doi:10.1103/PhysRevLett.119.241601.

70. Gould, O.; Mangl. S.; Rajantie, A.; Rose, S.; Xie, C. Observing Thermal Schwinger Pair Production Phys. Rev. A 2019, 99, 052120, doi:10.1103/PhysRevA.99.052120

71. Baines, S.; Mavromatos, N.E.; Mitsou, V.A.; Pinfold, J.L.; Santra, A. Monopole production via photon fusion and Drell-Yan processes: MadGraph implementation and perturbativity via velocity-dependent coupling and magnetic moment as novel features. Eur. Phys. J. C, 2018, 78, 966, doi:10.1140/epjc/s10052-018-6440-6.

72. Santra, A. Production of Magnetic Monopoles via Photon Fusion—Implementation in MadGraph. Proceedings 2019, 13, 4, doi:10.3390/proceedings2019013004.

73. Baines, S. Effective Field Theory Treatment of Monopole Production by Drell-Yan and Photon Fusion for Various Spins. MDPI Proc. 2019, 13, 1, doi:10.3390/proceedings2019013001.

74. CDF Collaboration. Direct search for Dirac magnetic monopoles in $p \bar{p}$ collisions at $\sqrt{s}=1.96 \mathrm{TeV}$. Phys. Rev. Lett. 2006, 96, 201801, doi:10.1103/PhysRevLett.96.201801.

75. Kalbfleisch, G.R.; Luo, W.; Milton, K.A.; Smith, E.H.; Strauss, M.G. Limits on production of magnetic monopoles utilizing samples from the D0 and CDF detectors at the Tevatron. Phys. Rev. D 2004, 69, 052002, doi:10.1103/PhysRevD.69.052002.

76. Price, P.B.; Ren, G.X.; Kinoshita, K. Search for Highly Ionizing Particles at the Fermilab Proton Anti-proton Collider. Phys. Rev. Lett. 1987, 59, 2523-2526, doi:10.1103/PhysRevLett.59.2523.

77. Aubert, B.; Musset, P.; Price, M.; Vialle, J.P. Search for Magnetic Monopoles in Proton-Anti-proton Interactions at 540-GeV Center-of-mass Energy. Phys. Lett. 1983, 120, 465-467, doi:10.1016/0370-2693(83)90488-4.

78. Carrigan, R.A.; Nezrick, F.A.; Strauss, B.P. Search for Magnetic Monopole Production by 300-GeV Protons. Phys. Rev. D 1973, 8, 3717-5720, doi:10.1103/PhysRevD.8.3717.

79. OPAL Collaboration. Search for Dirac magnetic monopoles in $e^{+} e^{-}$collisions with the OPAL detector at LEP2. Phys. Lett. B 2008, 663, 37-42, doi:10.1016/j.physletb.2008.03.057.

80. Pinfold, J.L.; Du, R.; Kinoshita, K.; Lorazo, B.; Regimbald, M.; Price, B. A Search for highly ionizing particles produced at the OPAL intersection point at LEP. Phys. Lett. B 1993, 316, 407-411, doi:10.1016/0370-2693(93)90346-J.

81. Kinoshita, K.; Du, R.; Giacomelli, G.; Patrizii, L.; Predieri, F.; Serra, P.; Spurio, M.; Pinfold, J.L. Search for highly ionizing particles in $e^{+} e^{-}$annihilations at $\sqrt{s}=91.1-\mathrm{GeV}$. Phys. Rev. D 1992, 46, R881-R884, doi:10.1103/PhysRevD.46.R881. 
82. Kinoshita, K.; Fujii, M.; Nakajima, K.; Price, P.B.; Tasaka, S. Search for Highly Ionizing Particles in $e^{+} e^{-}$Annihilations at $\sqrt{s}=50-\mathrm{GeV}$ to $60.8-\mathrm{GeV}$. Phys. Lett. B 1989, 228, 543-547, doi:10.1016/0370-2693(89)90990-8.

83. Musset, P.; Price, M.; Lohrmann, E. Search for Magnetic Monopoles in Electron - Positron Collisions at 34-GeV Center-of-mass Energy. Phys. Lett. 1983, 128, 333-335, doi:10.1016/0370-2693(83)90270-8.

84. CLEO Collaboration. Search for magnetically charged particles produced in $e^{+} e^{-}$annihilations at $\sqrt{s}=10.6 \mathrm{GeV}$. Phys. Rev. D 1987, 35, 1081-1084, doi:10.1103/PhysRevD.35.1081.

85. TASSO Collaboration. A Search for Particles With Magnetic Charge Produced in $e^{+} e^{-}$Annihilations at $\sqrt{s}=35 \mathrm{GeV}$. Z. Phys. C 1988, 38, 543-550, doi:10.1007/BF01624358.

86. ATLAS Collaboration. The ATLAS Experiment at the CERN Large Hadron Collider. JINST 2008, 3, S08003, doi:10.1088/1748-0221/3/08/S08003.

87. ATLAS Collaboration. Search for magnetic monopoles in $\sqrt{s}=7 \mathrm{TeV} p p$ collisions with the ATLAS detector. Phys. Rev. Lett. 2012, 109, 261803, doi:10.1103/PhysRevLett.109.261803.

88. ATLAS Collaboration. Search for magnetic monopoles and stable particles with high electric charges in $8 \mathrm{TeV} p p$ Collisions ATLAS Detector. Phys. Rev. D 2016, 93, 052009, doi:10.1103/PhysRevD.93.052009.

89. Akesson, T.; Arik, E.; Assamagan, K.; Baker, K.; Barberio, E.; Barberis, D.; Bertelsen, H.; Bytchkov, V.; Callahan, J.; Catinaccio, A.; Danielsson, H.; et al. Particle identification using the time-over-threshold method in the ATLAS Transition Radiation Tracker. Nucl. Instrum. Methods A 2001, 474, 172-187, doi:10.1016/S0168-9002(01)00878-6.

90. ATLAS Collaboration. Performance of the ATLAS Transition Radiation Tracker in Run 1 of the LHC: Tracker properties. JINST 2017, 12, P05002, doi:10.1088/1748-0221/12/05/P05002.

91. ATLAS Collaboration. Search for magnetic monopoles and stable high-electric-charge objects in $13 \mathrm{TeV}$ proton-proton collisions with the ATLAS detector. arXiv 2019, arXiv:1905.10130.

92. MoEDAL Collaboration. Search for magnetic monopoles with the MoEDAL forward trapping detector in $2.11 \mathrm{fb}^{-1} 13 \mathrm{TeV}$ proton-proton collisions at the LHC. Phys. Lett. B 2018, 782, 510-516, doi:10.1016/j.physletb.2018.05.069.

93. MoEDAL Collaboration. Technical Design Report of the MoEDAL Experiment; CERN-LHCC-2009-006; MoEDAL-TDR-001; 2009. Available online: http://inspirehep.net/record/1299494/ (accessed on 15 November 2018).

94. MoEDAL. Available online: http:/ / moedal.web.cern.ch/ (accessed on 15 November 2018).

95. Pinfold, J.L. The MoEDAL Experiment at the LHC-A Progress Report from the High Energy Frontier. Universe 2019, 5, 47, doi:10.3390/universe5020047.

96. DeRoeck, A.; Katre, A.; Mermod, P.; Milstead, D.; Sloan, T. Sensitivity of LHC Experiments to Exotic Highly Ionising Particles. Eur. Phys. J. C 2012, 72, 1985, doi:10.1140/epjc/s10052-012-1985-2.

97. MoEDAL Collaboration. The Physics Programme of the MoEDAL Experiment at the LHC. Int. J. Mod. Phys. A 2014, 29, 1430050, doi:10.1142/S0217751X14300506.

98. Fairbairn, M.; Kraan, A.C.; Milstead, D.A.; Sjöstrand, T.; Skands, P.; Sloan, T. Stable massive particles at colliders. Phys. Rept. 2007, 438, 1-63, doi:10.1016/j.physrep.2006.10.002.

99. Burdin, S.; Fairbairn, M.; Mermod, P.; Milstead, D.; Pinfold, J.; Sloan, T.; Taylor, W. Non-collider searches for stable massive particles. Phys. Rept. 2015, 582, 1-52, doi:10.1016/j.physrep.2015.03.004.

100. DeRoeck, A.; Hächler, H.P.; Hirt, A.M.; Joergensen, M.-D.; Katre, A.; Mermod, P.; Milstead, D.; Sloan, T. Development of a magnetometer-based search strategy for stopped monopoles at the Large Hadron Collider. Eur. Phys. J. C 2012, 72, 2212, doi:10.1140/epjc/s10052-012-2212-x.

101. MoEDAL Collaboration. Search for magnetic monopoles with the MoEDAL prototype trapping detector in $8 \mathrm{TeV}$ proton-proton collisions at the LHC. JHEP 2016, 1608, 067, doi:10.1007/JHEP08(2016)067.

102. MoEDAL Collaboration. Search for magnetic monopoles with the MoEDAL forward trapping detector in $13 \mathrm{TeV}$ proton-proton collisions at the LHC. Phys. Rev. Lett. 2017, 118, 061801, doi:10.1103/PhysRevLett.118.061801. 
103. MoEDAL Collaboration. Magnetic monopole search with the full MoEDAL trapping detector in $13 \mathrm{TeV}$ $p p$ collisions interpreted in photon-fusion and Drell-Yan production. Phys. Rev. Lett. 2019, 123, 021802, doi:10.1103/PhysRevLett.123.021802.

104. CMS Beam Pipe to be Mined for Monopoles. CERN Courier, 8 March 2019, p. 8. Available online: https: / / cerncourier.com/cms-beam-pipe-to-be-mined-for-monopoles/ (accessed on 1 April 2019).

(C) 2019 by the author. Licensee MDPI, Basel, Switzerland. This article is an open access article distributed under the terms and conditions of the Creative Commons Attribution (CC BY) license (http:/ / creativecommons.org/licenses/by/4.0/). 